\title{
RÉDACTION SPÉCIALISÉE ET UTILISATION DU DICTIONMAIRE
}

\author{
Jacqueline Bossë-Andrieu
}

Les dictionnaires sont les principaux outils du rédacteur. Malheureusement, les étudiants que nous formons à la rédaction spécialisée utilisent trop peu le dictionnaire ou bien, contrairement à ce qu'ils pensent, s'en servent mal. C'est pourquoi, à l'École de traducteurs et d'interprètes de l'Universitè d'ottawa, dañs les cours où est enseignée la rédaction de documents professionnels, 1 nous jugeons essentiel de démontrer aux étudiants qu'ils ne savent pas lire le dictionnaire. Ce qui ne va pas sans mal. Il faut des trēsors de patience pour prouver à l'étudiant qui vous rétorque "c'est dans le dictionnaire" ou "c'est ce que dit le dictionnaire" qu'il n'est pas donné à tout le monde de savoir lire un dictionnaire et que, même si on vérifie tous les mots, on n'est pas sûr d'aboutir à un texte convenable. En effet, ce n'est pas parce qu'un mot est consigné dans les dictionnaires qu'on sait d'emblée l'employer en discours; il faut savoir tirer parti des remarques incluses dan l'article, soupçonner que ce dernier est incomplet et, enfin, interprēter correctement les renvois. Ce qui, finalement, n'est pas aussi facile qu'on pourrait le croire. Mne J. Rey-Debove n'a-t-elle pas écrit: "En poussant les choses à l'extrême, seuls les auteurs d'un dictionnaire sont vraiment en mesure d'en tirer profit" ? ${ }^{2}$ Et il $\mathrm{m}^{\prime a}$ semblé intéressant d'examiner de plus près la nature des difficultés éprouvées à ce point de vue par mes étudiants.

En premier lieu, il faut signaler que les étudiants 1) ont en gënēral une confiance aveugle dans le dictionnaire qu'ils ont sous la main (sans même se douter que la date de parution est importante); 2) ignorent qu'il existe divers dictionnaires de langue générale--conçus dans des optiques différentes et que chacun a ses avantages, et de multiples dictionnaires spécialisēs; 3) doivent apprendre que ce n'est pas parce que l'un répond à la plupart de leurs besoins quotidiens que la consultation des autres est superflue, et que c'est en comparant ce que disent plusieurs qu'ils acquerront une meilleure idée de l'emploi de tel mot dans une phrase donnée.

En deuxième lieu, il convient, semble-t-il, de distinguer deux types d'obstacles sur lesquels butent les apprentis-rëdacteurs; il y a ceux qui sont dûs au dictionnaire lui-mème et qui sont inhérents à sa fabrication, et ceux qui relèvent plus précisément du contexte canadien.

Un premier piège dans lequel tombe plus d'un esprit non averti: les coquilies que contient n'importe quel ouvrage d'imprimerie. 3 Pourquoi, en effet, celui que le rëdacteur considère comme son ami le plus fidèle ne serait-il pas exempt de telles failles? Ce genre de constatation facilite en un sens la tâche de l'enseignant qui peut démontrer noir sur blanc qu'il faut parfois se méfier de ce que dit le 
dictionnaire, et ainsi dessiller les yeux des plus incrédules: de telles évidences permettent d'ébranler la confiance de ceux qui ont le tort de ne pas douter assez souvent. Effectivement, l'un des principaux reproches que le professeur de traduction ou de rédaction adresse à ses étudiants est de partir trop souvent du principe que ce qu'ils écrivent est idiomatique et bien français. Il est vrai que certaines recherches peuvent ne rien révēler à celui qui ne sait pas lire et qui ne sait pas qu'un dictionnaire ne dit pas tout--loin de là--et qu'il faut savoir deviner ce qui'il ne dit pas. De l'avis même d'une spécialiste des dictionnaires de langue que nous avons déjà citée, Mme Rey-Debove, "le dictionnaire est [...] par définition l'ouvrage où l'on trouve ce qu'on ne cherche pas et oú $l^{\prime}$ 'on ne trouve pas ce que l'on cherche (et ceci est vrai non seulement des dictionnaires de langue, mais de tous les dictionnaires)."4 Pour prendre un exemple parmi tant d'autres, je citerai le cas du syntagme "porter attention à quelque chose" relevé dans un grand nombre de textes rëdigés par mes étudiants. La plupart des dictionnaires indiquent implicitement par leurs exemples quels sont les syntagmes usuels, mais ils ne signalent évidement pas les combinaisons impossibles. Ainsi, aucun des dictionnaires courants, qui ne mentionnent $\mathfrak{a}$ l'article attention que les groupements faire attention et prêter attention (à quelque chose), ne permet de soupçonner que $\mathrm{T}$ 'on ne peut impunement substituer porter à prêter; en effet, avec porter la syntaxe du verbe change et on doit faire précéder le substantif dun adjectif possessif et le faire suivre d'une préposition différente. On aura ainsi porter son attention sur quelque chose (qui a d'ailleurs un sens lëgèrement différent, porter signifiant ici "diriger"). Pourquoi, demande l'étudiant? Et le professeur ne peut que répondre: "Parce que c'est comme ça!" C'est effectivement l'usage qui décide que, bien que porter amitié, intërêt, assistance, secours, envie (à quelqu'un) se disent, Ta combinaison de porter et d'attention sans dēterminant n'est pas acceptable. 5 Même si la limite entre "suite de mots fréquente mais modifiable" et "suite de mots intangible" 6 est imprécise, il faut savoir que, l'usage étant capricieux, il y a des associations obligatoires, permises ou défendues. Tant que les dictionnaires usuels ne seront pas plus systématiques en ce qui concerne les contraintes syntaxiques, 7 le rédacteur devra être constamment sur ses gardes. Mentionnons en passant que c'est une des lacunes qu'entend réparer le Dictionnaire explicatif et combinatoire du français contemporain (DECFC); ce dictionnaire de type nouveau élaboré à l'Universite de Montréal, "vise à décrire tous les lexèmes que le dictionnaire contient de façon aussi complète que possible avec 1 'accent sur la cooccurrence syntaxique et lexicale".8 II répondra aux besoins de ceux qui cherchent "des informations sur le rëgime syntaxique d'un mot et les façons concrètes de l'associer à ses compléments" et sur "la capacitē d'un mot de former des locutions figées avec d'autres." 9

Non seulement les dictionnaires génēraux ne donnent pas systématiquement toutes les constructions dans lesquelles peut entrer un mot, mais ils ne donnent pas non plus explicitement tous les sens des mots, même de ceux qui sont abondamment traités. Par exemple, essayant 
d'approfondir la différence qui séparait l'emploi du nom admission en français standard et en français canadien, je suis restée perplexe devant les définitions aussi bien du Petit Robert que du Lexis. D'abord, pour le verbe admettre, le premier donne comme cinquième sens: "Laisser entrer. "D'inextrícables ruelles qui ne peuvent admettre de voitures" (Gauthier)"10; et le deuxième "Admettre un être animé: le laisser entrer dans un lieu, dans un groupe ou une organisation: Admettre des yoyageurs en surnombre. Les chiens ne sont pas admis dans le magasin."11 Quant au substantif, le Petit Robert le definit comme "action d'admettre, fait d'être admis" et le Lexis, qui l'a placé sous 1 'entrëe admettre, ne donne aucune définition et se borne à citer des exemples. Comment expliquer alors qu'une expression telle que Pas d'admission au sens de "Défense d'entrer" constitue un anglicisme 12 si admission est l'action d'admettre un être animé? D'autant plus que Lexis, đans sa préface note: "Á 1 'intérieur de l'article, il n'a pas paru nécessaire de dēfinir les dërivēs dont le sens se déduisait exactement de celui du mot-souche. Cependant, si le dérivé présente une déviation, une restriction ou une extension de sens par rapport au mot-souche, la définition est toujours explicitée."13 Rien donc dans ces dictionnaires ne laisserait soupçonner que la phrase "l'admission des chiens dans le magasin est interdite" est inacceptable en français général. Et pourtant, si l'on étudie attentivement, dans les deux dictionnaires cités, les exemples contenant le substantif admission, on constate que dans aucun d'entre eux ce mot n'est employẻ au sens de "fait d'admettre temporairement dans un 1 ieu", sens que l'on était en droit de déduire logiquement de la définition du verbe. Le substantif $n^{\prime}$ a donc pas toute 1 'extension du verbe. Mais c'est une déduction qui n'est pas évidente. Seule une lecture minutieuse des exemples et des synonymes peut nous mettre la puce à l'oreille...et encore.

Et ce n'est pas le seul type de situation où les dictionnaires manquent de clarté. En plus des cas courants (définitions plus ou moins tautologiques, ou encore nuances sēparant des quasi-synonymes difficiles à saisir), citons celui où les définitions sont de nature à brouiller les idées du rédacteur. Ainsi, à l'article grâce du Petit Robert, il est donné comme définition du troisième sens: Pardon. 10(XIIIe). Pardon, remise de peine, de dette accordēe bénévolement. V. Amnistie, sursis.(...) \#(Sans article) Demander grâce. V. Misëricorde, quartier. Crier grâce. Voir supplier." Comme ce dernier sens de "grâce" est regroupe sous "Pardon", même si le losange indique une nuance de sens ou d'emploi à l'intérieur d'un sens, il est tentant de considérer 1 'expression demander grâce comme synonyme de demander pardon, ce qui est erroné et engendre impropriētēs et contresens. ${ }^{14}$ II faut donc interpréter les définitions avec beaucoup de circonspection. Il faut aussi être pénétré du fait qu'un dictionnaire courant (je ne parle donc pas ici d'ouvrages comme le Trésor de la langue française ou le Grand Robert) ne peut être exhaustif et contenir toutes les nuances de sens et tous les synonymes possibles du mot vedette. Comment pourrait-il en effet inclure tous les sens qu'un mot peut prendre en discours puisque "la définition du dictionnaire d'usage ampute le sens de sa dimension 
paradigmatique, en ignorant les rapports qu'entretient le mot avec les mots du micro-système auquel il appartient" et "que le sens d'un mot en contexte tient à la fois "aux autres mots présents dans l'énoncé" et "aux mots absents, mais évoqués plus ou moins prëcisẻment dans le projet d'énoncé". 15

Les dictionnaires ne contiennent pas non plus tous les mots contemporains, pour la bonne raison qu'ils ont toujours un certain retard par rapport à l'usage qui doit d'abord consacrer un mot avant que celui-ci fasse partie de leur nomenclature. C'est un problème particulièrement aigu au Canada où, en raison de l'insécurité linguistique qui caractérise la plupart des francophones, ces derniers ont trop souvent tendance à penser que, puisqu'un mot ne figure pas dans la dernière ëdition de leur dictionnaire, ce mot n'est pas "français". Il est normal que des mots correspondant à des réalités nouvelles $n$ 'entrent pas en même temps dans la langue et dans les dictionnaires (ainsi, le mot douchette qui désigne le système installé sur les éviers et comprenant une petite pomme d'arrosoir, que l'anglais appelle spray hose, ne se trouve pas encore dans le Petit Larousse alors qu'i est utitisé par les quincailliers européens). Néanmoins on peut juger plus ētrange que des mots abondamment employés dans la presse francophone--ils constituent souvent, il est vrai, des néologismes de sens--soient absents des dictionnaires. Exemples: rares sont les dictionnaires de langue qui donnent à holocauste le sens si fréquent de nos jours de "massacre massif de personnes", 10 sens qui ne figure que depuis 1984 dans le Petit Larousse. De mëme, le terme autosuffisance, création qui date des années 70 , ne se trouve dans aucun ouvrage courant. $17^{q}$ Ce qui explique l'hésitation de certains à l'employer. Le rédacteur sūr de sa langue ne pensera sans doute même pas à vérifier ce mot, mais celui qui doute et prend la peine de le faire--ne serait-ce que pour s'assurer de la graphie (emploi ou non du trait d'union)18.court le risque de se priver d'un mot fort utile.

Au Canada français, la peur de commettre un anglicisme peut priver les francophones--tout au moins dans la langue écrite--de certains termes pourtant en usage dans d'autres pays de la francophonie. Nous pensons à un terme comme coupe-vent employé au sens d'anorak léger; bien que, pour l'instant, le seulsens "standard" de coupe-vent reconnu par les dictionnaires soit celui de "dispositif de tôte placé à l'avant des vëhicules rapides pour rēduire la résistance de l'air", "I9 et qu'au sens anglais de wind-breaker il soit condamné ici comme étant un anglicisme, 20 il figure dans certains catalogues européens 21 et s'emploie parfois dans la langue parlée. Que faire au Canada, sinon s'armer de patience, en attendant que le sens de ce mot acquière outre--Atlantique ses lettres de noblesse et soit donné par les dictionnaires de l'Hexagone sans être accompagné de la mention "régionalisme," comme c'est le cas à I'heure actuelle dans le Petit Robert où on lit: "Rēg. (Canada; d'apr, l'amér. wind-breaker). Blouson dont Te tissue protège contre le vent." 22 
Ces remarques nous amènent à parler d'un dernier type de difficultés rencontrées par le rédacteur francophone canadien ou par le traducteur anglophone qui doit traduire vers l'anglais un texte écrit en français. C'est celui des canadianismes que ne mentionne pour ainsi dire aucun ouvrage. Prenons l'exemple de l'expression "à toute(s) fin(s) pratique(s)," très courante ici, en particulier dans la bouche des hommes politiques et sous la plume des journalistes. Celui qui, par acquit de conscience, veut vérifier s'il doit employer le singulier ou le pluriel aura la surprise de découvrir que cette locution ne figure dans aucun dictionnaire du français standard, la seule construction rēpertoriēe étant "à toutes fins utiles", qui ne correspond pas du tout à "à toute(s) fin(s) pratique(s)."23 Que faire encore en pareil cas? La prudence devrait conseiller au rédacteur de s'abstenir de l'employer et de chercher un synonyme, et au traducteur de dégager le sens de l'expression uniquement d'après le contexte. Tant qu'il n'existera pas de dictionnaire canadien répertoriant systématiquement tous les mots de la langue franco-canadienne et explicitant une norme, les rédacteurs hésiteront à employer certains mots et les écarteront pour la seule raison qu'ils ne figurent pas au Petit Robert. Mais c'est là un problème qui déborde notre propos; il montre cependant qu'il est encore plus difficile à un rédacteur canadien qu'à un rédacteur parisien de déjouer les chausses-trappes de son dictionnaire.

Qu'importe-t-il donc d'apprendre, à cet égard, à l'étudiant que nous préparons à la rédaction spécialisée? Á mon sens, il faut essentiellement lui apprendre à douter de tout, à douter non seulement de lui mais aussi "du" dictionnaire. S'il doute de lui, il consultera fréquemment son dictionnaire préfëré et évitera ainsi peut-être certaines impropriétés puisqu'il se rendra compte que le premier mot qui lui vient à l'esprit n'est pas toujours le bon. S'il parvient à douter "du" dictionnaire, il apprendra la prudence--mais c'est une habitude qui ne s'acquiert pas toujours rapidement étant donné que les dictionnaires sont les ouvrages de référence par excellence et qu'il est difficile de mettre en doute l'infaillibilité de ce dont on vous a toujours vanté la perfection. Finalement l'étudiant saura qu'il faut lire chaque article avec des lunettes (mentales) grossissantes qui lui permettront de tenir compte de toutes les remarques, y compris les remarques d'usage (telles que $v x$, vieilli, litt., techn., rêq., etc.) qu'il a trop souvent tendance a négliger; il prendra conscience du fait qu'il importe aussi de lire les renseignements donnés à travers le prisme de son expérience, de son savoir linguistique, de sa logique et qu'il est souvent nécessaire de pousser ses recherches plus loin et de ne pas s'arrêter à la consultation d'un seul dictionnaire puisque la réponse à certaines des questions qu'il se pose se trouve vraisemblablement dans des ouvrages conçus dans une optique différente. Si cette rëflexion d'un 
linguiste vise essentiellement les dictionnaires bilingues, nous croyons qu'elle corrobore notre propos:

Pourquoi un dictionnaire, imparfait et souvent peu systēmatique, est-il utilisable pour 1 'homme et inutilisable pour la machine? La seule réponse possible est que l'homme--même une personne peu intelligente--dispose de connaissances dont la machine ne dispose pas et qui, s'ajoutant aux informations contenues dans le dictionnaire, lui permettent de profiter de ces informations imparfaites et souvent peu systématiques. 24

Il est essentiel de convaincre nos étudiants de la nécessité de s'aider de leurs connaissances pour lire un dictionnaire et savoir interpréter ce qu'il dit. Les dictionnaires sont des aides indispensables, car nul ne peut connaitre la langue à la perfection, et ils sont d'un immense secours lorsque--comme dans le cas des béquilles-on a appris à s'en servir.

\section{NOTES}

1. $\dot{A}$ l'École de traducteurs et d'interprètes de l'Universitè d'Ottawa, certains cours du programme de traduction, communs à ceux du programme de certificat en rédaction française et en rédaction anglaise, prëparent nos ètudiants à devenir rédacteurs bilingues (et unilingues). Rappelons que le rédacteur bilingue prépare un texte en langue $A$ à partir d'un texte rëdigé en langue $B$ (sans pour autant avoir recours à la traduction), ou à partir de documents donnés en langue $B$.

2. Josette Rey-Debove, Étude linguistique et sémiotique des dictionnaires français contemporains, Paris-La Haye, Mouton, 1971, p. 313.

3. Il suffit de mentionner ici deux coquilles relevēes, la première, dans le Petit Robert (1984)--qui sera désormais abrégé en P.R., et la deuxiême dans Te Lexis (1977)--désormais abrégé en LX. Ainsi dans le PR, la particule vice est donnée comme invariable (plus loin on lit "des vice-chanceliers," "des vice-légats," "des vice-prēsidents," "des vice-rois"), mais l'entrée vice-consul est suivie de la mention du pluriel: "vices-consuls". Dans le LX, on lit, à l'article "principe," la dëfinition suivante de de principe: "qui ne concerne que l'essentiel: dont les dēlais [sic]n'ont pas èté réglēs: Un accord de principe". C'est sans doute "détails" que l'on voulait dire, comme Te confirme le Grand Larousse encyclopëdique: "De principe, qui ne concerne que l'essentiel; dont les détails $n$ 'ont pas été réglés: Un accord de principe.

4. Josette Rey-Debove, op. cit., p. 39. 
5. C'est ici que la consultation d'ouvrages d'un genre différent est utile: le Dictionnaire des mots et des idées de U. Lacroix, qui réunit autour de substantifs les verbes et adjectifs qui s'emploient éventuellement avec eux, donne ainsi pour attention: "Attirer, éveiller, appeler, exciter [...] l'attention. Fixer, concentrer, porter, détourner son attention. [...] L'attention s'éveille, se fixe, se porte."

6. Alain Rey, Prëface du P.R., p. XV.

7. En 1981, dans "La lexicographie française: rétrospective et perspectives," Alain Rey proposait quelques directions souhaitables pour la lexicographie française, dont "une prise en considération plus systématique des contraintes syntactiques et de leur intrication avec la semantique, notamment pour les verbes et ies adjectifs." (Lexique 2. Le dictionnaire, p. 23.)

8. Betty Cohen et Lẻo Elnitsky, "Un nouveau type de dictionnaire utile aux traducteurs: le OEC du français moderne," in META, vol. 29, no. 2 , juin 1984 , p. 159 .

9. Ibid.

10. P.R. (1984), P. 26.

11. $L X(1979)$, p. 28 .

12. Voir Colpron, Dictionnaire des anglicismes, p. 4 ou encore Le Dictionnaire des difficultés de la langue française au Canada de Gérard Dagenais, pp. 14-15.

13. $L X(1979)$, P. XI.

14. Je cite cet exemple, car j'ai eu toutes les peines du monde à convaincre de leur erreur un groupe d'étudiants qui soutenaient avec opiniâtreté que le P.R. disait que demander grâce, c'était demander pardon, alors qu'en fait cette expression signifie "se déclarer vaincu, à bout de forces" ( $L X)$ et donc implorer la pitié du vainqueur ou de celui devant lequel on déclare forfait.

15. Robert Galisson, Lexicologie et enseignement des langues (essais méthodologiques), paris, Hachette, 1979, p. 130 .

Voir aussi a ce sujet l'article de Solange Chevrier-Vouvé, "Les connecteurs pragmatiques, l'analyse de discours et la traduction," in Bulletin de l'ACLA, automne 1984, vol. 6, no. 2, pp. 147-58. Reprenant une analyse de Marianne Schelling de l'université de Genève portant sur les "conclusifs"--mots qui expriment ou indiquent une conclusion--, l'auteur montre que le traducteur/rédacteur revient bredouille de sa chasse au sens quand il tente de trouver dans les dictionnaires le sens de au fond connecteur, c'est-à-dire employé pour articuler un texte $\overline{\text { et pour }}$ introduire "une conclusion qui n'était pas nécessairement prévue au début de l'échange par le locuteur lui-même" (op. cit., p. 157). 
16. Cette extension de sens se trouve néanmoins dans le TLF et le Dictionnaire du français vivant.

17. Il figure cependant dans le Dictionnaire des mots contemporains de Pierre Gilbert, mais ne fait pas T'objet d'une entrée séparée; il est simplement donné à l'entrée auto, parmi les nombreux composēs formés à partir de cet èlément.

18. En fait, le Dictionnaire des mot contemporains donne les deux graphies, avec ou sans trait d'union, bien que cette dernière soit à notre avis devenue plus courante.

19. Petit Larousse 1984.

20. Voir en particulier le Dictionnaire des difficultēs de la langue française au Canada de Gérard Dagenais qui assène: Coupe-vent au sens de blouson est un mauvais calque de l'anglais wind-breaker, dont la traduction littérale serait plutōt brise-vent" lop. cit., p. 166). G. Colpron (op. cit., p. 29) classe aussi ce terme parmi les anglicismes en mentionnant toutefois que, dans le bulletin C'est-à-dire, le Comité linguistique de Radio-Canada le donne comme néologisme au sens d'anorak sans doublure, en nylon, avec capuchon qu'on enfile par-dessus la tête et garni souvent d'une poche kangourou sur le devant."

21. Coupe-vent figure dējà dans ce sens dans le catalogue des TroisSuisses, hiver 80-81, pp. 104 et 310 .

22. Est-ce à dire qu'il serait prēférable, aux yeux de certains, d'employer $K$-Way, qui a l'avantage de figurer au P.R. 84,

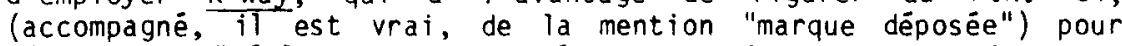
désigner la "légère veste imperméable en nylon qui se replie dans une poche qu'on peut attacher à la ceinture"? Mais gardons-nous de ranimer ici la vieille querelle de "shopping"/"magasinage" ou de "week-end"/"fin de semaine"!

23. En effet, d'après le P.R., à toutes fins utiles signifie "pour servir le cas échèant et en tout cas". Quant à à toutes fins pratiques qui figure néanmoins dans le Dictionnaire nord-américain de la langue française de Bëlisle (sans être donné comme canadianisme), il semble que ce soit l'équivalent de "à tout prendre," "dans la pratique" (Bēlisle), et même de "en fait," "pour ainsi dire," "en somme," "au fond." Il y aurait donc dans "à toute(s) fin(s) pratique(s)" tēlescopage de l'expression "à toutes fins utiles" et de l'adverbe "pratiquement" auquel a ètē emprunté le sens néologique (et critiqué) de "presque," "à peu de chose près."

24. Bondi Sciari, "Analyse sémantique: I'homme et la machine," in Lexique 2. Le Dictionnaire, op. cit., p. 133. 


\section{RÉFÉRENCES}

Bēlisle, Louis-Alexandre, Dictionnaire nord-amëricain de la langue frança ise, Montréal, Beauchemin, 1979.

Chevrier-Vouvé, Solange, "Les connecteurs pragmatiques, l'analyse du discours et la traduction," in Bulletin de 1 'ACLA, automne 1984, vol. 6, no. 2, pp. 147-58.

Cohen, Betty, et Lēo Elnitsky, "Un nouveau type de dictionnaire utile aux traducteurs: le DEC du français moderne," in META, vol. 29, no. 2, juin 1984, pp. 159-75.

Colpron, Gilles, Dictionnaire des anglicismes, Montréal, Beauchemin, 1982.

Dagenais, Gérard, Dictionnaire des difficultēs de la langue française au Canada, Boucherville, les Editions françaises, 1984 /2e édition).

Davau, M., M. Cohen, M. Lallemand, Dictionnaire du français vivarit, Paris, Bordas, 1976.

Galisson, Robert, Lexicologie et enseignement des langues (essais méthodologiques), Paris, Hachette, 1979.

Gilbert, Pierre, Dictionnaire des mots contemporains, "les usuels du Robert", Paris, Le Robert, 1980.

Lacroix, U., Dictionnaire des mots et des idées, Paris, Nathan, 1969.

$\frac{\text { Le Petit Larousse illustré, dictionnaire encyclopëdique pour tous, }}{\text { Paris, Larousse, } 1984 \text {. }}$

Le Petit Robert 1, Dictionnaire alphabétique et analogique de la langue française, Paris, Le Robert, 1984.

Lexis, Paris, Larousse, 1979.

Rey, Alain, "La lexicographie française: rētrospective et perspectives," in Lexique 2. Le dictionnaire, Actes du colloque franco-néerlandais, 28-29 avril 1981, Presses universitaires de Lille, 1981.

Rey-Debove, Josette, Étude linguistique et sëmiotique des dictionnaires français contemporains, Paris, La Haye, Mouton, 1971. 
Sciari, Bondi, "Analyse sëmantique: I'homme et la machine," in Lexique 2. Le dictionnaire, Actes du colloque franco-nēerlandais, 28-29 avril 1981, Presses universitaires de Lille, 1981 .

Trésor de la langue française, Tome neuvième, Paris, Éditions du centre nationa $T$ de la recherche scientifique, 1981.

Jacqueline Bossë-Andrieu est professeur adjoint à l'Ecole des traducteurs et d'interprètes, Université d'ottawa. 